\title{
Finding the condition of Turing instabilities
}

\author{
Turing's diffusive threshold in random reaction-diffusion systems \\ Authors: Pierre A. Haas, and Raymond E. Goldstein \\ bioRxiv:2020.11.09.374934v1

\begin{abstract}
Recommended with a Commentary by Changbong Hyeon, Korea Institute for Advanced Study
\end{abstract}

As exemplified in leopard's coat and zebra stripe, temporally stable and spatially inhomogeneous steady state patterns are ubiquitous in the animal body [1]. Manifestation of such patterns is also a fundamental basis of embryogenesis, which constitutes a series of collective symmetry-breaking events among effectively identical cells at the early stage of development. In his seminal paper entitled "The chemical basis of morphogenesis," Alan Turing put forward a simple reaction-diffusion based mechanism, widely known as the diffusion-induced instability or Turing instability, to account for the formation of biological patterns [2]. Inhomogeneous steady state concentration profiles of diffusible signaling molecules, termed 'morphogens,' was hypothesized to induce differential gene expressions across a collection of cells and formation of biological patterns. Specifically, Turing proposed that interplay between two types of morphogens, autocatalytic activators and inhibitors, diffusing across the cells, can self-organize to generate spatial inhomogeneous periodic concentration profiles of the morphogens.

In general terms without resorting to a specific model, the spatiotemporal dynamics of $N$-interacting morphogens is explained using the reaction-diffusion equation with an appropriate boundary condition, $\dot{\mathbf{c}}=\mathbf{f}(\mathbf{c})+\mathbf{D} \cdot \nabla^{2} \mathbf{c}$, where $\mathbf{c}$ is the concentrations of morphogens, $\mathbf{c}=\mathbf{c}(\mathbf{r}, t) \equiv\left(c_{1}(\mathbf{r}, t), c_{2}(\mathbf{r}, t), \ldots c_{N}(\mathbf{r}, t)\right), \mathbf{f}(\mathbf{c}) \equiv\left(f_{1}(\mathbf{c}), f_{2}(\mathbf{c}), \ldots, f_{N}(\mathbf{c})\right)$ represents a set of nonlinear functions of the concentrations $\mathbf{c}$, and $\mathbf{D}$ is the diffusion matrix. The dynamical properties of the solution $c(\mathbf{r}, t)$ at steady states are assessed by performing a stability analysis of a linearized equation around the fixed point $\mathbf{c}^{*}$ that satisfies $\mathbf{f}\left(\mathbf{c}^{*}\right)=\mathbf{0}$. At $\mathbf{c} \simeq \mathbf{c}^{*}+\delta \mathbf{c}$ $\left(\left|\delta c_{i} / c_{i}^{*}\right| \ll 1\right.$ for all $\left.i\right)$, the equation is linearized to:

$$
\partial_{t} \delta \mathbf{c}=\mathbf{J} \cdot \delta \mathbf{c}+\mathbf{D} \cdot \nabla^{2} \delta \mathbf{c}
$$

where $\mathbf{J}$ is the Jacobian matrix evaluated at $\mathbf{c}=\mathbf{c}^{*}$. It is expected that the solution $\delta \mathbf{c}(r, t)$ is obtained as the sum of Fourier modes,

$$
\delta \mathbf{c}(\mathbf{r}, t) \sim \sum_{k} \phi_{k} e^{\lambda\left(k^{2}\right) t} e^{i \mathbf{k} \cdot \mathbf{r}}
$$

where $\phi_{k}$ is the coefficient of each Fourier mode, and $\lambda=\lambda\left(k^{2}\right)$ is the roots of characteristic polynomial of degree $N$,

$$
\operatorname{det}\left(\lambda \mathbf{I}-\mathbf{J}+k^{2} \mathbf{D}\right)=0 .
$$


(i) The temporal stability is ensured for $k=0$ when all the roots of the characteristic polynomial $\operatorname{det}[\lambda \mathbf{I}-\mathbf{J}]=0$ have negative real parts $\left(\operatorname{Re}\left(\lambda_{i}\right)<0\right.$ for all $\left.i\right)$. (ii) The Turing instability leading to a spatially inhomogeneous, periodic steady state pattern arises when at least one of the roots has positive real part giving rise to unstable modes of wavenumber $k$. The fluctuation in morphogen concentration that grows exponentially with time is eventually balanced with the nonlinear terms in the equations [3].

As shown by Haas and Goldstein [4], for $N=2$ with $\mathbf{J}=\left(\begin{array}{ll}f_{u} & f_{v} \\ g_{u} & g_{v}\end{array}\right)$ and $\mathbf{D}=\left(\begin{array}{cc}d_{u} & 0 \\ 0 & d_{v}\end{array}\right)$, the conditions for temporal stability and Turing instability are straightforwardly determined from the quadratic polynomial of $\lambda$ as

$$
\begin{aligned}
& f_{u}+g_{v}<0 \\
& \operatorname{det} \mathbf{J}>0 \\
& d_{v} f_{u}+d_{u} g_{v}>0 \\
& \left(d_{v} f_{u}+d_{u} g_{v}\right)^{2}-4 d_{u} d_{v} \operatorname{det} \mathbf{J}>0
\end{aligned}
$$

with det $\mathbf{J}=f_{u} g_{v}-f_{v} g_{u}$. It is immediately clear (from Eqs.4 and 6) that the diffusivities of two morphogens should differ from each other $\left(d_{u} \neq d_{v}\right)$ for Turing instability, and the Jacobian matrix is also constrained to have a structure, such that $f_{v} g_{u}<f_{u} g_{v}<0$, i.e., $\mathbf{J}=\left(\begin{array}{ll}+ & + \\ - & -\end{array}\right),\left(\begin{array}{ll}+ & - \\ + & -\end{array}\right),\left(\begin{array}{ll}- & - \\ + & +\end{array}\right),\left(\begin{array}{ll}- & + \\ - & +\end{array}\right)$. Lastly, Eq.7 provides an inequality that the ratio of two diffusivities, $D_{2}=\max \left(d_{v} / d_{u}, d_{u} / d_{v}\right)$ should satisfy, specifying the threshold value $D_{2}^{*}$,

$$
D_{2}>D_{2}^{*}= \begin{cases}\left(\frac{\sqrt{\operatorname{det} \mathbf{J}}+\sqrt{\operatorname{det} \mathbf{J}-f_{u} g_{v}}}{f_{u}}\right)^{2} & \text { for } d_{v} / d_{u}>1 \\ \left(\frac{\sqrt{\operatorname{det} \mathbf{J}}+\sqrt{\operatorname{det} \mathbf{J}-f_{u} g_{v}}}{g_{v}}\right)^{2} & \text { for } d_{u} / d_{v}>1\end{cases}
$$

For given values of the four elements of Jacobian matrix $\left(f_{u}, f_{v}, g_{u}, g_{v}\right)$, which defines $R=\frac{\max \left(\left|f_{u}\right|,\left|f_{v}\right|,\left|g_{u}\right|,\left|g_{v}\right|\right)}{\min \left(\left|f_{u}\right|,\left|f_{v}\right|,\left|g_{u}\right|,\left|g_{v}\right|\right)}$, the diffusive threshold $D_{2}^{*}$ ranges over $R \leq D_{2}^{*} \leq D_{2}^{\max }(R)$ with $D_{2}^{\max }(R)\left[=\left(R+\sqrt{\left(R^{2}-1\right)}\right)^{2}\right]$. By using randomly generated Jacobian matrices, Haas and Goldstein showed that the probability of $D_{2}^{*}$ smaller than $R$ is low $\left(P\left(D_{2}^{*} \leq R\right)=\right.$ $\left.1-\int_{R}^{D_{2}^{\max }} P\left(D_{2}^{*}\right) d D_{2}^{*} \approx 0.03\right)$, indicating that the occurrence of Turing instability is unlikely for the case of $(N=2)$-species systems if the diffusivities of two morphogens are similar [4]. Given that molecules with similar sizes are characterized with similar diffusivities, at least for the case of 2-species morphogen systems the Turing instability or diffusion-induced instability does not seem to be a viable mechanism to explain the origin of biological patterns.

The same type of stability/instability analysis shown above can be repeated for $N=3$, but with substantially increased algebraic complexity [4]. Without explicitly solving the roots of polynomial, we can benefit from Routh-Hurwitz stability criterion that help us judge whether all the roots of polynomials lie in the left half plane $(\operatorname{Re}(\lambda)<0)$. The temporal stability condition (i.e., $\operatorname{Re}\left(\lambda_{i}\right)<0$ for all $i$ ) for cubic polynomials $(N=3)$, 
$\lambda^{3}+p_{1}\left(k^{2}\right) \lambda^{2}+p_{2}\left(k^{2}\right) \lambda+p_{3}\left(k^{2}\right)=0$, is acquired if and only if $p_{1}(0)>0, p_{3}(0)>0$, and $p_{1}(0) p_{2}(0)-p_{3}(0)>0$. For instability to occur and form spatial patterns, any of the $k$ dependent inequalities, i.e., $p_{1}\left(k^{2}\right)>0, p_{3}\left(k^{2}\right)>0$, and $p_{1}\left(k^{2}\right) p_{2}\left(k^{2}\right)-p_{3}\left(k^{2}\right)>0$, should be violated over some range of $k[5,6]$. For $N=4, \lambda^{4}+p_{1} \lambda^{3}+p_{2} \lambda^{2}+p_{3} \lambda+p_{4}=0$, the stability condition is $p_{1}>0, p_{1} p_{2}-p_{3}>0,\left(p_{1} p_{2}-p_{3}\right) p_{3}-p_{1}^{2} p_{4}>0$, and $p_{4}>0$. An analysis may still be doable, but the algebraic complexity in identifying the stability condition increases rapidly with $N$.

Incidentally, studying the condition for Turing pattern formation in $\mathrm{N}$-species systems with $N \gg 1$ is in essence mathematically equivalent to assessing the stability of large dynamic systems comprised of many interacting components. In 1970s, Gardner and Ashby [7] studied the stability of general dynamic systems by representing the corresponding Jacobian matrix (A) with $C$ percent of 'connectance' (non-zero elements chosen from $\mathcal{N}(0,1)$ ) using random matrices. Namely, they assessed the stability of $\mathbf{x}(t)$ obeying the linear equation,

$$
d \mathbf{x} / d t=\mathbf{A} \cdot \mathbf{x}
$$

by means of the numerical calculation of eigenvalues of randomly generated $\mathbf{A}$. For such systems with size $N$ to be stable, all the eigenvalues must have the negative real parts. They discovered that for $N \geq 10$ and $C \approx 13 \%$, A matrices almost always have at least one eigenvalue with non-negative real part, suggesting that the systems characterized with $13 \%$ connectance undergoes a sharp transition from stable to unstable dynamic behaviors at $N=10$. May [8] later generalized Gardner and Ashby's numerics-based conjecture and provided the stability criterion explicitly based on Wigner's random matrix theory with an additional parameter, an average interaction strength $\alpha$, the variance of random numbers to be assigned to generate a random matrix,

$$
\alpha<\frac{1}{(N C)^{1 / 2}} .
$$

This stability criterion suggests that weighted by the extent of connectance $(C)$ there is a critical size of the system, $N_{c}\left(=1 /\left(\alpha^{2} C\right)\right)$ beyond which the system displays instability.

Motivated by May's stability analysis for random matrix-based complex system, Haas and Goldstein carried out exercises of generating $N \times N$ random Jacobian matrices $\mathbf{J}$ from $N=2$ to $N=6$ and numerically evaluating the value of diffusive threshold $D_{N}^{*}$ from the discriminant obtained for $N$. They found that the probability of random $\mathbf{J}$ having a Turing instability, $P\left(D_{N}^{*} \leq R\right)$, increases with $N$ as well as $R$ and that the diffusive threshold is gradually lowered with increasing $N$.

Although it has long remained elusive since the Turing's proposal, recent experimental demonstrations on biological pattern formation via diffusible signaling molecules seem to lend support to Turing mechanism $[9,10]$. Given that there are many molecular players during the animal development, Haas and Goldstein's theoretical work leading to the conclusion on the reduction of diffusive threshold due to many morphogen species makes the diffusion-induced mechanism of Turing instabilities more plausible.

\section{References}

[1] Murray, J. D, Scientific American 258, 80 (1988). 
[2] Turing, A. M, Phil. Trans. Roy. Soc. London B, Biol. Sci. 237, 37 (1952).

[3] Murray, J, Mathematical Biology II: spatial models and biomedical applications, SpringerVerlag, 2001.

[4] Haas, P. A \& Goldstein, R. E, bioRxiv , 10.1101/2020.11.09.374934 (2020).

[5] Kuznetsov, M \& Polezhaev, A, Chaos 30, 033106 (2020).

[6] Anma, A, Sakamoto, K, \& Yoneda, T, Kodai Mathematical Journal 35, 215 (2012).

[7] Gardner, M. R \& Ashby, W. R, Nature 228, 784 (1970).

[8] May, R. M, Nature 238, 413 (1972).

[9] Raspopovic, J, Marcon, L, Russo, L, \& Sharpe, J, Science 345, 566 (2014).

[10] Di Patti, F, Lavacchi, L, Arbel-Goren, R, Schein-Lubomirsky, L, Fanelli, D, \& Stavans, J, PLoS biology 16, e2004877 (2018). 